\title{
Evolutionary Status and Internal Structure of $\mu$ Cassiopeiae
}

\author{
KIEHUNN BACH \\ Department of Astronomy, Yonsei University, 50 Yonsei-ro, Seodaemun, Seoul, Korea; kbach@yonsei.ac.kr
}

Received February 11, 2015; accepted March 20, 2015

\begin{abstract}
We investigate physical properties of the nearby $(\sim 7.5 \mathrm{pc})$ astrometric binary $\mu$ Cas in the context of standard evolutionary theory. Based on the spectroscopically determined relative abundances $([\alpha / \mathrm{Fe}] \gtrsim+0.4 \mathrm{dex},[\mathrm{Fe} / \mathrm{H}] \sim-0.7 \mathrm{dex})$, all physical inputs such as opacities and equation of state are consistently generated. By combining recent spectroscopic analyses with the astrometric observations from the HIPPARCOS parallaxes and the CHARA array, the evolutionary model grids have been constructed. Through the statistical evaluation of the $\chi^{2}$-minimization among alternative models, we find a reliable evolutionary solution $\left(M_{A}, M_{B}, t_{\text {age }}\right)=\left(0.74 \mathrm{M}_{\odot}, 0.19 \mathrm{M}_{\odot}, 11 \mathrm{Gyr}\right)$ which excellently satisfies observational constraints. In particular, we find that the helium abundance of $\mu$ Cas is comparable with the primordial helium contents $\left(Y_{p} \sim 0.245\right)$. On the basis of the well-defined stellar parameters of the primary star, the internal structure and the $p$-mode frequencies have been estimated. From our seismic computation, $\mu$ Cas is expected to have a first order spacing $\Delta \nu \sim 169 \mu \mathrm{Hz}$. The ultimate goal of this study is to describe physical processes inside a low-mass star through a complete modelling from the spectroscopic observation to the evolutionary computation.
\end{abstract}

Key words: stars: individual: $\mu$ Cas — fundamental parameters — evolution — interiors — atmospheres — asteroseismology

\section{INTRODUCTION}

Accurate determination of physical parameters is a crucial cornerstone in stellar astrophysics. Especially, stellar mass is the most fundamental factor with which the lifetime of a star is outlined. In principle, an important application of binary systems is that they provide the most reliable method to measure stellar masses. In this respect, with the well-defined parallaxes through the orbital analysis, nearby visual binaries are invaluable contributors to define physical parameters of stars. Moreover, the chemical abundances of the old stellar populations provide essential constraints in our understanding of the stellar evolution. Recent development of high-resolution spectroscopic observations has greatly enhanced our ability to reconstruct the galactic chemical enrichment history. In this study, we investigate physical parameters of a well-constrained visual binary through a consistent stellar modelling. Especially, we focus primarily on the evolutionary status and internal structure of this system through the calibration of stellar parameters.

The visual binary $\mu$ Cassiopeiae ( $\mu$ Cas, HD6582, HR321, HIP5336, GJ53) is a low-mass old Population star in the solar neighborhood. Owing to its proximity $(\sim 7.5 \mathrm{pc})$ and brightness $\left(M_{V} \sim 5.17\right)$, the orbital elements have been well-defined through a series of astrometric observations (Russell \& Gatewood 1984; Haywood et al. 1992; Drummond et al. 1995). This binary system consists of G5 + M5 pair of main-sequence stars with the orbital period $(P \sim 22 \mathrm{yr})$. However, in spite of the well-constrained total mass $\left(\Sigma M \sim 0.9 \mathrm{M}_{\odot}\right)$,

Corresponding AUthor: K. Bach a large magnitude difference $(\Delta \mathrm{mag} \sim 6.5)$ makes it hard to determine each individual mass of components. Employing the full adaptive optics, Drummond et al. (1995) estimated the mass ratio of $\left(M_{A}, M_{B}\right) \sim(0.75$ $\left.\mathrm{M}_{\odot}, 0.17 \mathrm{M}_{\odot}\right)$. More recently, Lebreton et al. (1999) provided an evolutionary solution based on the more accurate HIPPARCOS parallax data. Most of all, as it is a very rare case in contemporary astrophysics, Boyajian et al. (2008) directly measured the radius of the primary star $\left(R \sim 0.79 R_{\odot}\right)$ using the optical interferometric observation of the CHARA array (Center for High Angular Resolution Astronomy). In general, radius is a fundamental stellar parameter which impacts on the internal structure and atmospheres. Especially, one of the most uncertain properties in the one-dimensional (1D) stellar model is the mixing length parameter $\left(\alpha_{M L T}\right)$ and the Eddington $T(\tau)$ relation as a boundary condition. Therefore, an accurate determination of stellar radii implies that these parameters can be evaluated using a well-defined observational constraints.

From its kinematics of a high space velocity $(\sim 160$ $\left.\mathrm{km} \mathrm{s}^{-1}\right)$ and metallicity $([\mathrm{Fe} / \mathrm{H}] \sim-0.7 \mathrm{dex}), \mu$ Cas has been categorized into halo stars (Hearnshaw 1974; Russell \& Gatewood 1984). The chemical compositions of late-type stars play a crucial role in improving our understanding of galactic and cosmic evolution. In this context, the chemical abundance of $\mu$ Cas has attracted the attention of many observers (Catchpole et al. 1967; Cohen 1968; Hearnshaw 1974). Recent high resolution spectroscopy permits the precise determination of relative abundances with respect to iron (Takeda 2007; Mishenina et al. 2013; Bach \& Kang 2015). Through their spectroscopic analysis of cool 
Table 1

Astrometric parameters of the $\mu$ Cas system

\begin{tabular}{|c|c|c|c|}
\hline Element & Lippincott (1981) & Russell \& Gatewood (1984) & Drummond et al. (1995) \\
\hline$P$ & $21.715 \pm 0.100 \mathrm{yr}$ & $21.855 \pm 0.100 \mathrm{yr}$ & $21.753 \pm 0.069 \mathrm{yr}$ \\
\hline$a$ & $0.1979 \pm 0 . .0074$ & $0^{\prime \prime} 1879 \pm 0^{\prime \prime} 0067$ & $0^{\prime \prime} 1908 \pm 0^{\prime \prime} 0043$ \\
\hline$e$ & $0.544 \pm 0.027$ & $0.525 \pm 0.025$ & $0.561 \pm 0.017$ \\
\hline$i$ & $106^{\circ} 2 \pm 1^{\circ} .1$ & $106^{\circ} 0 \pm 1.1$ & $106.8 \pm 0.9$ \\
\hline$\Omega$ & $47^{\circ} 8 \pm 1.4$ & $48.4 \pm 1.3$ & $47^{\circ} .3 \pm 1.0$ \\
\hline$\omega$ & $334^{\circ} .3 \pm 4.6$ & $336^{\circ} 3 \pm 4.7$ & $332^{\circ} .7 \pm 3.1$ \\
\hline$\pi$ & $0 . .132 \pm 0 . \prime 002$ & $0^{\prime \prime} 1386 \pm 0^{\prime \prime} .0033$ & $0^{\prime \prime} 1333 \pm 0^{\prime \prime} .0017$ \\
\hline$\Sigma M / \mathrm{M}_{\odot}$ & $0.972 \pm 0.079$ & $0.879 \pm 0.084$ & $0.916 \pm 0.058$ \\
\hline$M_{A} / \mathrm{M}_{\odot}$ & $0.783 \pm 0.080$ & $0.718 \pm 0.086$ & $0.742 \pm 0.059$ \\
\hline$M_{B} / \mathrm{M}_{\odot}$ & $0.189 \pm 0.015$ & $0.161 \pm 0.015$ & $0.173 \pm 0.011$ \\
\hline
\end{tabular}

stars, the relative abundances of $\mu$ Cas have been determined to be $[\mathrm{Mg} / \mathrm{Fe}] \sim+0.43$ dex (Zhao \& Gehren $2000),[\mathrm{C} / \mathrm{Fe}] \sim+0.4$ and $[\mathrm{O} / \mathrm{Fe}] \sim+0.5$ (Takeda \& Honda 2005; Mishenina et al. 2013) suggesting that the $\alpha$-captured element is enhanced. On the other hand, Dennis (1965) suggested that $\mu$ Cas is a favorable source from which the primordial helium abundance can be measured using stellar evolutionary theory. Therefore, $\mu$ Cas has significance for the chemical composition of the elemental enhancement and the helium abundance. Combining the astrometric orbit analysis and the evolutionary model computations, Haywood et al. (1992) obtained the helium abundance of this star $(0.18 \leq Y \leq 0.23)$. However, a proper treatment of gravitational sedimentation of helium was not considered in their computations, so their estimation is too lower than the recent measurements of the primordial helium content $\left(Y_{p} \sim 0.245\right)$.

In this study, we investigate physical properties of the $\mu$ Cas binary system utilizing the recent high-resolution spectroscopy of the BOES observation (Bach \& Kang 2015). By collecting recent spectrophotometric observations, stellar parameters have been defined in the context of standard stellar theory. In our evolutionary computation, physical quantities that govern the stellar interior are consistently generated based on the chemical mixture. Through the statistical evaluation, we find a reliable evolutionary solution of $\mu$ Cas which is in excellent agreement with the recent observational constraints. Regarding the microscopic diffusion process, we also examine an initial helium abundance of this system. In addition, using the best fit model, the $p$-mode frequencies of the primary star are estimated within the framework of asteroseismology. The ultimate goal of the present paper is: (1) to constrain the physical dimensions through a consistent modelling in the context of standard stellar theory, (2) to provide an estimation of a possible frequency spectrum of the mode oscillation, and (3) to produce a reliable initial configurations for the three-dimensional radiation-hydrodynamic simulations for low-mass stars.

\section{OBSERVATIONS}

\subsection{Fundamental Parameters}

Since Wagman (1961) discovered the binarity of $\mu$ Cas from the variable proper motion, a series of astrometric observations of the parallax and orbital parameters has been continued. In earlier studies, the position angle and the separation of the system were measured by double-aperture interferometry (Wickes \& Dicke 1974; Wickes 1975) and by narrow-band photography (Feibelman 1976). Through compilation of several decades of photometry, this nearby $(\sim 7.5 \mathrm{pc})$ binary system is found to have a priod of $P \sim 22$ years (Wagman et al. 1963; Lippincott \& Wyckoff 1964; Lippincott 1981; Russell \& Gatewood 1984). Spectroscopically, $\mu$ Cas is categorized as a single-line binary consisting of a G5 + M5 pair of subdwarf stars with low metallicity. Based on the spectroscopic orbit analysis, Worek \& Beardsley (1977) also provided a similar period and orbital elements. However, a large magnitude difference $(\Delta$ mag $\sim 6.5)$ between components make it hard to resolve a faint companion, $\mu$ Cas B. More recently, Drummond et al. (1995) determined the astrometric orbit and relative masses combining his adaptive optics observations with the previous astrometric measurements (Lippincott 1981; Russell \& Gatewood 1984). The recent orbital elements and mass ratios of the $\mu$ Cas system are summarized in Table 1.

The radius of a star is one of the most fundamental characteristics that is tightly related to the internal structure and evolutionary status. Conventionally, a star approximates well to a point source located at an infinite distance. In this respect, it has been believed that a direct detection of stellar radii is nearly impossible to achieve. Generally, the radius of a star has been indirectly estimated by relying on the mass-luminosity relation of stellar models. Fortunately, recent advances in the long-baseline optical interferometry enable us to directly measure stellar angular diameters of nearby stars (Lane et al. 2001; Ségransan et al. 2003; Berger et al. 2006). In a recent interferometric observation using the CHARA array, Boyajian et al. (2008) determined the angular diameter of the primary component $\mu$ Cas A with a high accuracy within a $1 \%$ error. Their radius 
Table 2

Physical properties of $\mu$ Cas

\begin{tabular}{lccl}
\hline Parameter & $\mu$ Cas A & $\mu$ Cas B & Ref. \\
\hline$T_{\text {eff }}(\mathrm{K})$ & $5297 \pm 32$ & $3034 \pm 160$ & $1-4,6,7$ \\
$\mathrm{M}_{b}$ & $5.54 \pm 0.04$ & $10.22 \pm 0.14$ & 2,4 \\
$M / \mathrm{M}_{\odot}$ & $0.742 \pm 0.059$ & $0.173 \pm 0.011$ & 2,4 \\
$L / \mathrm{L}_{\odot}$ & $0.442 \pm 0.014$ & $0.0062 \pm 0.0008$ & $1,2,4$ \\
$R / \mathrm{R}_{\odot}$ & $0.791 \pm 0.008$ & $0.29 \pm 0.02$ & 2,4 \\
{$[\mathrm{M} / \mathrm{H}]$} & $-0.810 \pm 0.1$ & - & $3,4,5,7$ \\
$\log g$ & $4.52 \pm 0.04$ & - & $3,4,6,7$ \\
\hline
\end{tabular}

Note. 1. McCarthy et al. (1993) 2. Drummond et al. (1995) 3. Fuhrmann (1998a) 4. Boyajian et al. (2008) 5. Thévenin \& Idiart (1999) 6. Fulbright (2000) 7. Takeda (2007)

detection yields a well-constrained physical parameters such as an effective temperature $\left(T_{\text {eff }} \sim 5300 \mathrm{~K}\right)$, luminosity $\left(L / L_{\odot} \sim 0.442\right)$, and radius $\left(R / R_{\odot} \sim 0.79\right)$, which is in good agreement with the global properties provided by Drummond et al. (1995). Recent determinations of physical parameters and global properties are collected in Table 2 .

\subsection{The Primordial Helium Abundance}

The formation of the elements and the initial mass fraction are key subjects for understanding the cosmological evolution. Especially, the primordial helium abundance $\left(Y_{p}\right)$ is an essential cosmological parameter which reflects the initial condition of the early stage of the Universe. Historically, Gamow (1948) proposed that an initial amount of heavier elements than hydrogen is a fundamental consequence of nucleosynthesis in a few minutes after hot big bang. After that, Peebles (1966) provided an estimate of the primordial helium abundance $\left(0.22 \lesssim Y_{p} \lesssim 0.26\right)$ based on a homogeneous and isotropic cosmological model (see also Peimbert \& Torres-Peimbert 1999). In general, the primordial helium abundance is estimated from metal-poor extragalactic H II regions (Steigman 2007). Recent values of the initial helium abundance are determined to be $Y_{p}=0.245 \pm 0.003$ by Peimbert et al. (2007) and $Y_{p}=0.256 \pm 0.005$ by Izotov \& Thuan (2010). Comprehensive reviews on the recent developments of the primordial helium abundance are provided in literatures (e.g., Steigman 2007; Weinberg 2008; Pagel 2009).

Meanwhile, the primordial helium abundance is also empirically determined by the old stellar systems on the basis of the chemical enrichment history. The main idea is that the chemical enrichment can be established statistically by determination of the helium-to-metal ratio

$$
\Delta Y / \Delta Z=\left(Y-Y_{p}\right) / Z \text {. }
$$

For instance, Ribas et al. (2000) introduced $Y_{p}=$ $0.225 \pm 0.013$ using the detached double-lined eclipsing binaries (EGs). Through their statistical analysis of the globular cluster (GC) NGC 6752, Cassisi et al. (2003) provided $Y_{p}=0.244 \pm 0.006$. Moreover, using the morphology of blue horizontal branch (HB) stars, Villanova et al. (2009) yielded $Y_{p}=0.245 \pm 0.012$. Due to its long lifetime, a low-mass old population star still stays on the main-sequence phase, consequently it also becomes a favorable source that can provide observational constraints of the primordial helium abundance. For this reason, Dennis (1965) suggested that $\mu$ Cas would be a promising candidate for measurement of the primordial helium abundance. Later, combining the astrometric analysis and the evolutionary model computation, Haywood et al. (1992) obtained the initial helium abundance of $\mu$ Cas $(0.18 \leq Y \leq 0.23)$. However, since a proper treatment of the microscopic elemental diffusion was not included in their model, their estimation is lower than the recent measurements $\left(Y_{p} \sim 0.245\right)$. In this study, we examine the initial helium content of $\mu$ Cas by adjusting a fine grid of $Y_{0}$ instead of the slope of the chemical enrichment (Equation 1) which is considered in the most of isochrone-fitting methods.

\subsection{Spectroscopic Observations}

The chemical abundances of a metal-poor star have the potential to inform our understanding of an environmental condition when the star forms. The $\alpha$ elements are built from nucleosynthesis of $\mathrm{He}$ nuclei via $\alpha$-capture process during various stellar evolutionary stages, and subsequently they are dispersed during supernova (SN) explosions (Bensby \& Feltzing 2006; Reddy et al. 2006). Recent analyses of the stellar abundances revealed that the majority of metal-poor stars with $[\mathrm{Fe} / \mathrm{H}] \sim-1.0$ dexhave an $\alpha$-enhanced chemical mixture $([\alpha / \mathrm{Fe}]=+0.4 \mathrm{dex})$. Because $\mu$ Cas is a metalpoor halo star in the vicinity of the Sun, its elemental abundances are expected to deliver invaluable information related to the chemical enrichment history of the Galaxy. Particularly, from a point of the ELS model (Eggen et al. 1962), it was believed that $\mu$ Cas was formed in an early phase of rapid collapse during formation of the Galactic disk, since its orbital eccentricity is considerably greater than those of the thin disk stars.

In earlier spectroscopic observations (Catchpole et al. 1967; Cohen 1968), it is known that $\mathrm{Mg}$, Si, Ca, and $\mathrm{Ti}$ are overabundant with respect to iron by a factor of 2 . The near-infrared $\mathrm{OH}$ line analysis revealed that oxygen is also enhanced with $[\mathrm{O} / \mathrm{Fe}] \sim+0.28$ (Israelian et al. 1998; Meléndez et al. 2001). From the photospheric CNO abundances analysis of metal-poor stars, Takeda \& Honda (2005) derived a more enhancement of $[\mathrm{C} / \mathrm{Fe}] \sim+0.44$ and $[\mathrm{O} / \mathrm{Fe}] \sim+0.53$. Recent spectroscopic studies on the Galactic disk substructure, the enhancement of the $s$-process and the $r$-process elements has been identified (Takeda 2007; Takeda \& TakadaHidai 2011; Mishenina et al. 2002, 2004, 2013). In addition, through atmospheric analysis utilizing the $\mathrm{Bo}$ hyunsan Optical Echelle Spectrograph (BOES; Kim et al. 2002), Bach \& Kang (2015) confirmed that $\mu$ Cas has the $\alpha$-enhanced chemical compositions $([\alpha / \mathrm{Fe}] \gtrsim+0.4$ dex). In Table 3 , recent elemental analyses of relative abundances are collected (Takeda 2007; Mishenina et al. 2013; Bach \& Kang 2015).

Recent studies on NLTE effects have revealed that over-ionization by UV photons plays an important role in stellar atmospheres especially of metal-poor 
Table 3

Recent spectroscopic relative abundances of $\mu$ Cas

\begin{tabular}{lrcc}
\hline \multirow{2}{*}{ Element } & \multicolumn{3}{c}{$[\mathrm{X} / \mathrm{Fe}]$} \\
\cline { 2 - 4 } & TAK2007 & MIS2013 & BK2014 \\
\hline $\mathrm{C}$ & ${ }^{a} 0.44$ & - & 0.45 \\
$\mathrm{O}$ & ${ }^{a} 0.53$ & 0.51 & - \\
$\mathrm{Na}$ & 0.02 & ${ }^{c} 0.10$ & 0.04 \\
$\mathrm{Mg}$ & 0.43 & ${ }^{d} 0.37$ & 0.48 \\
$\mathrm{Al}$ & 0.29 & ${ }^{c} 0.38$ & 0.30 \\
$\mathrm{Si}$ & 0.23 & ${ }^{d} 0.32$ & 0.29 \\
$\mathrm{~S}$ & ${ }^{b} 0.22$ & - & - \\
$\mathrm{Ca}$ & 0.26 & 0.32 & 0.20 \\
$\mathrm{Sc}$ & 0.11 & - & 0.38 \\
$\mathrm{Ti}$ & 0.35 & - & 0.33 \\
$\mathrm{~V}$ & 0.24 & - & 0.13 \\
$\mathrm{Cr}$ & 0.02 & - & 0.00 \\
$\mathrm{Mn}$ & -0.12 & - & -0.26 \\
$\mathrm{Co}$ & 0.10 & - & 0.17 \\
$\mathrm{Ni}$ & 0.00 & ${ }^{d} 0.00$ & 0.06 \\
$\mathrm{Cu}$ & -0.06 & -0.03 & - \\
$\mathrm{Zn}$ & 0.30 & ${ }^{c} 0.25$ & 0.28 \\
$\mathrm{Y}$ & - & 0.04 & - \\
$\mathrm{Ba}$ & - & -0.12 & 0.27 \\
$\mathrm{La}$ & - & 0.10 & - \\
$\mathrm{Ce}$ & - & -0.06 & - \\
$\mathrm{Nd}$ & - & 0.13 & - \\
$\mathrm{Sm}$ & - & 0.19 & - \\
$\mathrm{Eu}$ & - & 0.41 & - \\
\hline
\end{tabular}

Note. TAK2007: Takeda (2007), MIS2013: Mishenina et al. (2013), BK2014: Bach \& Kang (2015), (a) Takeda \& Honda (2005), (b) Takeda \& Takada-Hidai (2011), (c) Mishenina et al. (2002), (d) Mishenina et al. (2004)

stars (Nissen et al. 1997; Fuhrmann 1998b; Feltzing \& Gustafsson 1998; Thévenin \& Idiart 1999). It is known that there is a serious discrepancy between spectroscopically determined surface gravities and those obtained from HIPPARCOS parallaxes (Nissen et al. 1997; Fuhrmann 1998a). Furthermore, Fulbright (2000) insisted that surface gravities deduced from HIPPAR$C O S$ are systematically overestimated. Through an extensive calculation of statistical ionization equilibrium, Thévenin \& Idiart (1999) quantitatively derived the NLTE effects for late-type stars. In cases of metalpoor main-sequence stars, Fe I line blocking decreases rapidly as the metal abundance decreases, and overionization becomes more important. Consequently, metallicity and surface gravity are significantly underestimated due to the over-ionization effect. In this study, we adopt their NLTE corrections: metallicity with $[\mathrm{Fe} / \mathrm{H}]_{\mathrm{NLTE}} \sim[\mathrm{Fe} / \mathrm{H}]_{\mathrm{LTE}}+0.15 \operatorname{dex}$ (Thévenin \& Idiart 1999) and surface gravity with $\log g_{\mathrm{NLTE}} \sim 4.52$ for the $\mu$ Cas system (Fulbright 2000).

\section{Evolutionary Status}

\subsection{Physical Inputs and Microscopic Diffusion}

Combining recent spectrophotometric observations, we investigate evolutionary status of the $\mu$ Cas binary system within the framework of the standard stellar the- ory. The evolutionary model grids are constructed using the Yale stellar evolution code (YREC) (Guenther et al. 1992) with recently updated physical inputs. In particular, opacities and equation of state (EOS) are crucial ingredients that have the serious impact on the stellar internal structure. Basically, two sets of the Rosseland mean opacities have been considered in our model computation: the OPAL opacities (Iglesias \& Rogers 1996) in the deep interior $\left(\log T_{\text {eff }} \geq 4.0\right)$ and low temperature opacities (Ferguson et al. 2005) near surface $\left(\log T_{\text {eff }} \leq 4.5\right)$. In the overlapping region $\left(4.0 \leq \log T_{\text {eff }} \leq 4.5\right)$, two opacities have been averaged. In case of equation of state, we adopt the OPAL EOS which is consistently computed with opacities. Above physical inputs are newly generated based on the $\alpha$-enhanced chemical composition with respect to the scaled solar abundance (Grevesse \& Sauval 1998). The GS98 solar mixture is known to be in good agreement with the sound-velocity distribution of the solar interior (Basu \& Antia 2008).

On the other hand, microscopic diffusion is a slow migration of small particles arising from gravitational settling and the thermal gradient inside stars. Feeding helium into the central region, the microscopic elemental diffusion can seriously modify the internal structure and surface constituents. Especially, in cases of low-mass stars, even a small rate of diffusion can significantly affect local physical properties (equation of state and opacities) during their long lifetime. Since the $\mu$ Cas system is composed of two cool stars, radiative levitation is practically negligible, so only gravitational sedimentation becomes important. In our evolutionary computation, both helium $(Y-)$ and metal $(Z-)$ diffusion are included on the basis of a standard description (Bahcall \& Loeb 1990; Thoul et al. 1994).

\subsection{Mixing Length}

In the outer envelope of solar-like stars, energy generated in the core is transported mainly by convection. Basically, convection itself is the three-dimensional (3D) phenomenon. In 1D model, the efficiency of the convective energy transport is usually prescribed by the classical mixing-length parameter (Böhm-Vitense 1958) defined as $\alpha_{\mathrm{MLT}}=l / H_{p}$. The super-adiabatic layers (SAL) of the stellar atmosphere are sensitive to the mixing-length parameter. Therefore, even a tiny variation of mixing length parameter can produce a significant change of the thermodynamic properties near surface. The recent standard solar model suggests that the mixing length parameter will be placed in the range between 1.5 and 2.0 (Cox \& Giuli 1968). Unfortunately, since the radius of a distant star cannot be accurately defined, a direct determination of mixing length parameter is believed to be mostly impossible. Most of all, to evaluate the mixing length parameters, stellar model atmosphere as a upper boundary condition should be defined priorly. For this reason, the solar value $\alpha_{\odot}$ (or a mild variation) has been unavoidably adopted in the 1D stellar model. This subject will be discussed further in Section 4. 

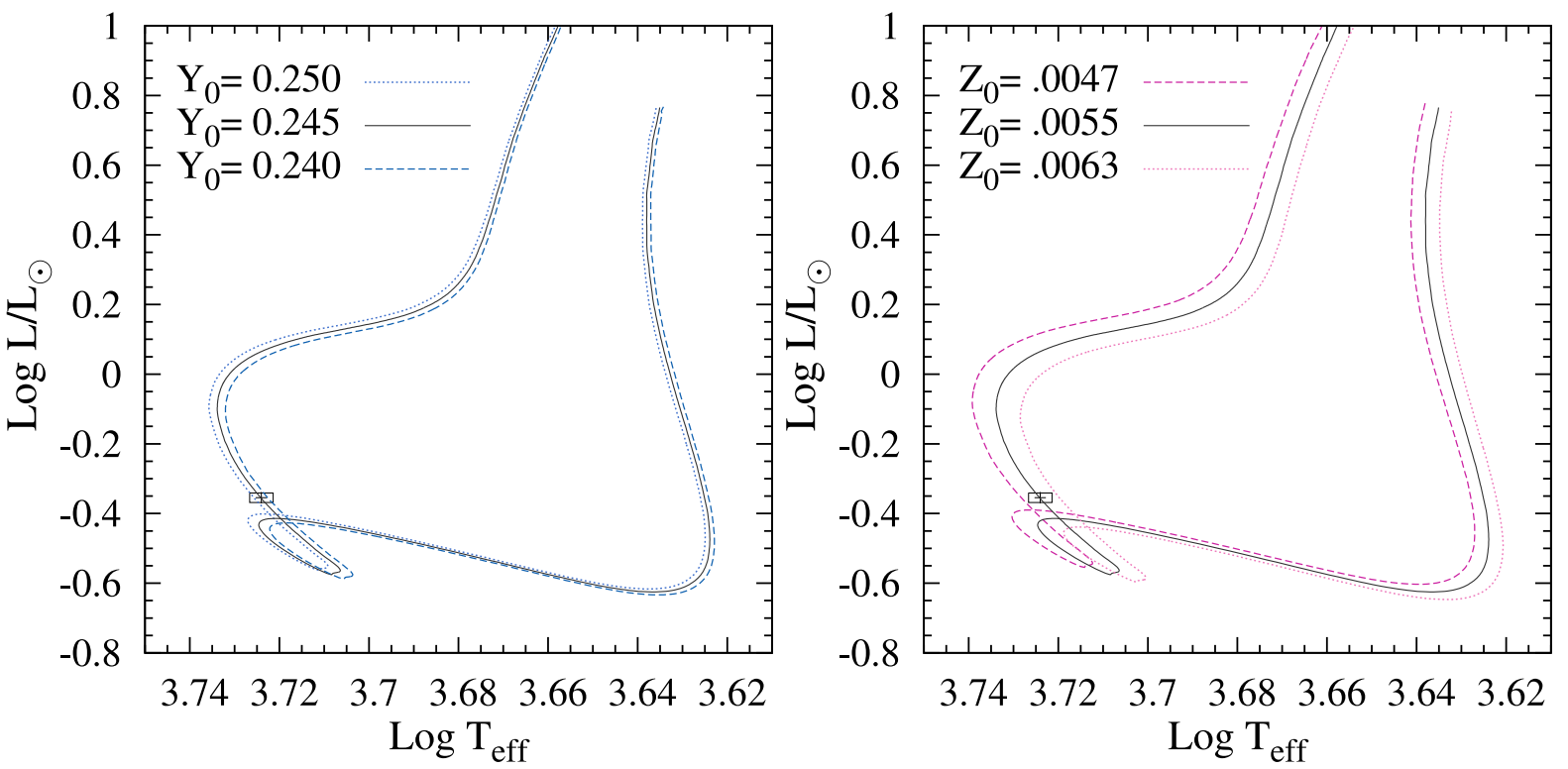

Figure 1. Evolution tracks of the $\mu$ Cas binary system. Through a statistical assessment, the best fit model (solid) which minimize the observational errors has been determined. By adjusting the initial helium abundance (left) and the metallicity (right), the representative models that marginally satisfy physical properties of the primary star have been presented. Boxes denote the domains of observational errors.

However, if physical dimensions of a stellar system are well-defined, the mixing-length parameter can be calibrated statistically (Fernandes et al. 1998). For instance, from the well-constrained stellar parameters of nearby detached binaries, a calibrated $\alpha_{\mathrm{MLT}}$ is in good agreement with those of the low-mass main-sequence slope of open clusters (Vandenberg \& Bridges 1984; Vandenberg \& Hrivnak 1985). Furthermore, it is known that the calibrated $\alpha_{\mathrm{MLT}}$ matches well to the effective temperature distribution of red giant branch (RGB) stars (Chieffi et al. 1995; Vandenberg \& Bridges 1984; Alonso et al. 1999). Most of all, recent advances in the optical interferometric observations enabled to directly detect the radius of the primary component $\mu$ Cas $\mathrm{A}$ (Boyajian et al. 2008). Therefore, our model grids include the mixing length calibration within a modest range of $\alpha_{\mathrm{MLT}}$. The solar mixing length parameter is calibrated to $\alpha_{\odot} \sim 1.88$ in our standard solar model based on the Grevesse \& Sauval (1998) abundances with $(Z / X)_{\odot} \sim 0.0229$. Here, metallicity of a star is defined as

$$
[\mathrm{M} / \mathrm{H}]=\log (Z / X)-\log (Z / X) \odot,
$$

where $X$ is the hydrogen mass fraction, and $Z$ is the mass fraction of metal elements at the stellar surface. If the mixing-length parameter is well-calibrated, then this classical approximation will provide a computationally efficient way of describing convection in the $1 \mathrm{D}$ stellar modelling.

\subsection{Calibration of Stellar Parameters}

In the context of the standard evolutionary theory, stellar models can be constructed from a set of initial configurations, that is, modelling parameters: stellar mass, metallicity, helium mass fraction, and the mix- ing length parameter. By adjusting modelling parameters, calibration of a star is to find an initial condition with which an evolved status satisfies observational constraints. In general, the standard evolutionary theory is valid for single stars. However, if a binary system is sufficiently detached and each component is within its Roche lobe, the standard evolutionary model can be safely applied. In this case, $\mu$ Cas is composed of two low-mass main-sequence stars $\left(M_{A}, M_{B}\right) \sim\left(0.7 \mathrm{M}_{\odot}\right.$, $\left.0.2 \mathrm{M}_{\odot}\right)$. From its subdwarf status and separation, we assume that there is no significant interaction between components without loss of generality. In addition, binary systems are supposed to have the same age $\left(t_{\text {age }}\right)$ with an identical initial chemical composition $\left(X_{0}, Y_{0}\right.$, $\left.Z_{0}\right)$. If the bright primary is calibrated with a wellconstrained observational errors, then, this calibrated configurations can be applied to the faint secondary star.

Based on the well-defined chemical mix (Table 3), we construct a model grids using the mass range $(0.10$ $\left.\mathrm{M}_{\odot} \leq M_{A} \leq 0.85 \mathrm{M}_{\odot}\right)$ with the step of $\Delta M=0.001$ $\mathrm{M}_{\odot}$. The initial mass fraction of metal element is assumed to be within the range $\left(0.001 \leq Z_{0} \leq 0.01\right)$ with the resolution of $\Delta Z=0.0001$. Regarding helium abundance as a free parameter with the constraint of $Y \gtrsim Y_{p}$, the initial helium content has been also examined in the range of $0.20 \leq Y_{0} \leq 0.30$ with $\Delta Y=0.005$. In addition, the mixing length parameter is estimated by regarding a mild variation $\left(\Delta \alpha_{\mathrm{MLT}}=0.01\right)$ with respect to the solar value $\left(\alpha_{\odot} \sim 1.88\right)$ that is obtained from the standard solar model. In our evolutionary computations, all models are computed from the beginning of the pre-main sequence (PMS) contraction, that is, the stellar birth-line, until the helium ignition occurs in the core. 
Table 4

Calibrated parameters and physical inputs

\begin{tabular}{lcc}
\hline & $\mu$ Cas A & $\mu$ Cas B \\
\hline$M / \mathrm{M}_{\odot}$ & $0.74 \pm 0.01$ & $0.19 \pm 0.04$ \\
$t_{\text {Age }}$ & $11.7 \pm 2.0 \mathrm{Gyr}$ \\
\hline$X_{0}$ & $0.7495 \pm 0.010$ \\
$Z_{0}$ & $0.0055 \pm 0.002$ \\
$Y_{0}$ & $0.2450 \pm 0.010$ \\
\hline$X_{s}$ & 0.8000 & 0.7414 \\
$Z_{s}$ & 0.0044 & 0.0055 \\
\hline$X_{c}$ & 0.2880 & 0.7406 \\
$Z_{c}$ & 0.0062 & 0.0055 \\
\hline$\alpha_{\mathrm{MLT}}$ & $1.65 \pm 0.2$ & $1.55 \pm 0.4$ \\
\hline$T_{\text {eff }}(\mathrm{K})$ & 5299 & 3136 \\
$L / \mathrm{L} \odot$ & 0.4418 & 0.0047 \\
$R / \mathrm{R} \odot$ & 0.7907 & 0.2238 \\
{$[\mathrm{M} / \mathrm{H}]$} & -0.62 & -0.50 \\
$\log g$ & 4.51 & 5.10 \\
\hline OPAL opacities & \\
OPAL equation of states \\
Low temperature opacities \\
Pheonix atmospheres \\
Microscopic $(Y-/ Z-)$ diffusion \\
\hline
\end{tabular}

Note. $\left(X_{0}, Y_{0}, Z_{0}\right)$ denotes the initial mass fraction at the beginning of the pre-main sequence contraction. $\left(X_{s}, Z_{s}\right)$ and $\left(X_{c}, Z_{c}\right)$ correspond to the mass fraction of hydrogen and metal elements at the surface, and in the central region, respectively.

From their statistical studies on kinematics and age of the solar neighborhood stars, Caloi et al. (1999) suggested that high space velocity stars $\left(V \sim 30 \mathrm{~km} \mathrm{~s}^{-1}\right)$ have a low metallicity $([\mathrm{Fe} / \mathrm{H}] \sim-0.6)$. Since $\mu$ Cas is categorized into a high-velocity star with respect to the local standard of rest, we also consider $t_{\text {age }} \geq 7 \mathrm{Gyr}$ as a constraint for the age estimation. In general, due to the many-fold degeneracy among physical parameters, the evolutionary solution of a star is not uniquely determined. For a statistical evaluation among possible models, the $\chi^{2}$-minimization (Lastennet et al. 1999) has been regarded:

$$
\chi^{2}=\sum\left[\left(\frac{A^{i}-A_{o b s}^{i}}{\sigma\left(A^{i}\right)}\right)^{2}\right],
$$

where $A^{i}$ denotes physical parameters of the theoretical model, $A_{o b s}^{i}$ is the center value of observation, and $\sigma(A)$ is the observational error of the corresponding quantities. In a conventional isochrone fit, age of the stellar system is generally estimated based on two physical properties $\left(L, T_{\text {eff }}\right)$ on the HR diagram. Instead, in order to minimize uncertainties between parameters, we consider more number of physical constraints $(L, R$, $\left.T_{\text {eff }}, \log g,[\mathrm{M} / \mathrm{H}]\right)$, which provides a better estimation of physical dimensions.

Through our evolutionary computations, we find a reliable set of physical parameters which statistically minimize observational errors. The calibrated parameters and global properties of the best fit model are listed

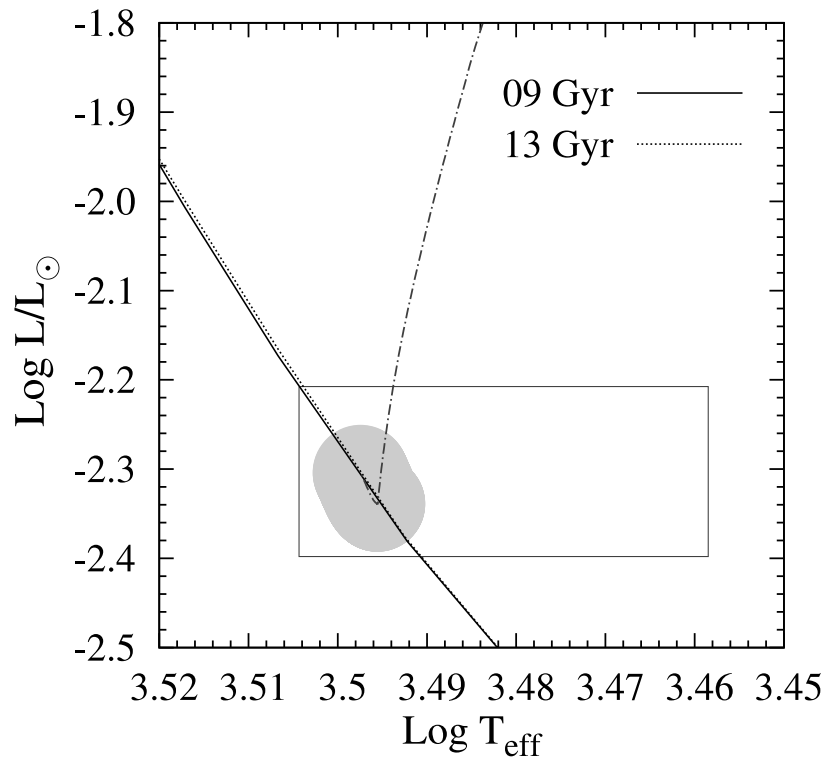

Figure 2. The evolution track of the secondary star. On the basis of the identical configurations of the primary star, the evolutionary status of $\mu$ Cas B has been calculated from the stellar birth-line to the age of $t_{\text {age }} \sim 11.7 \mathrm{Gyr}$. Two isochrones of $9 \mathrm{Gyr}($ solid) and $13 \mathrm{Gyr}($ dotted $)$ have been compared with the evolution track of $\mu$ Cas B on the HR diagram. The box denotes the observational error domain. The grey area corresponds to an alternative solution computed with uncertainties of the initial helium abundance $(\Delta Y=0.005)$, the fractional mass of metal elements $(\Delta Z=0.002)$, and mass $\left(\Delta M=0.02 \mathrm{M}_{\odot}\right)$.

in Table 4. From its relative abundances, metallicity and high space velocity, $\mu$ Cas seems to have a typical old population star. Accordingly, its evolutionary status has been estimated to be a main-sequence phase with an age of $t_{\text {age }} \sim 11$ Gyr. The evolutionary tracks of the primary star are presented in Figure 1. Most of all, it is notable that $\mu$ Cas has an extremely low helium abundance $\left(Y_{O} \sim 0.245\right)$ which is comparable to the primordial helium content $\left(Y_{p}\right)$. In the left panel of Figure 1, two representative models that have the different helium abundance $\left(Y_{0}=0.24,0.25\right)$ are compared with the best fit solution (solid). In the stellar structure theory, helium content has impact on the equation of state of internal gas by increasing the mean molecular weight. Half of the gravitational energy raises the temperature of the central region, and consequently it increases the nuclear reaction rates. Then, the outer layers expand compensating for the steepened temperature gradients in the central regions. Eventually, the larger mean molecular weight increases the luminosity of the star. On the other hand, with their low ionization potential, metal elements are the main opacity source by increasing the electron density. Therefore, the higher metallicity decreases the effective temperature and luminosity of the star. By changing the mass fraction of metal elements, the evolutionary tracks of two comparative models $\left(Z_{0}=0.0047,0.0055\right)$ are compared with the best fit model in the right panel of Figure 1.

Using the identical initial configurations of the pri- 


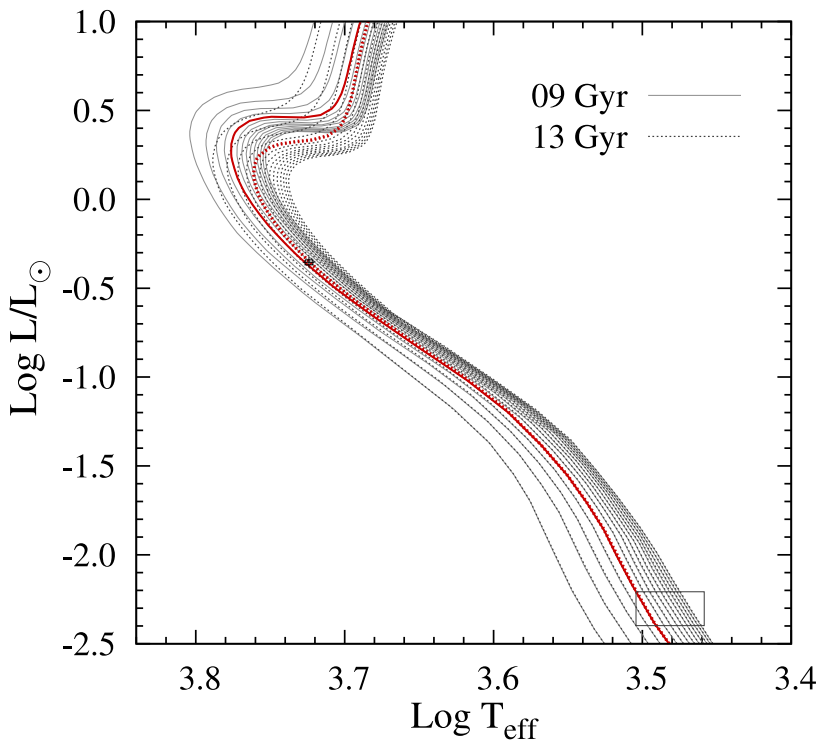

Figure 3. The age estimation of the $\mu$ Cas binary system. Theoretical isochrones of 9 Gyr (solid) and 13 Gyr (dotted) are presented on the HR diagram. According to the calibrated parameters from the primary star, isochrones are generated with the metallicity range $(0.002 \leq Z \leq 0.30)$ and the mass range $\left(0.1 \mathrm{M}_{\odot} \leq M \leq 2 \mathrm{M}_{\odot}\right)$. Boxes denote the observational error domains of each component.

mary, the faint secondary is calibrated to be a very low-mass star $\left(M_{B} \sim 0.19\right)$. Due to its long mainsequence lifetime, $\mu$ Cas $\mathrm{B}$ is slightly moved from the ZAMS position on the HR diagram. In Figure 2, the evolutionary track (dash-dot) of the secondary is presented with two isochrones (9 Gyr and $13 \mathrm{Gyr}$ ). The grey area corresponds to the location of an alternative model with uncertainties of the initial helium abundance $(\Delta Y=0.005)$, metallicity $(\Delta Z=0.002)$, and mass $\left(\Delta M=0.02 \mathrm{M}_{\odot}\right)$. In addition, the $\alpha$-enhanced isochrones are constructed on the basis of our model grids. In Figure 3, theoretical isochrones are illustrated with observational constraints of both components of the $\mu$ Cas system. Through a consistent modelling, our calibrations are in excellent agreement with the recent observational constraints (McCarthy et al. 1993; Drummond et al. 1995).

\subsection{Asteroseismic Analysis}

Asteroseismology is a unique tool for probes of stellar interiors. In general, acoustic waves can penetrate to different depths inside the star according to their oscillation modes. The mode frequencies provide the density profile of the internal region where the sound waves are emanating and travelling. Conversely, the oscillation modes can be translated into an estimation of the internal structure of each evolutionary phase. Owing to its detectable micro-variability, the primary component of $\mu$ Cas may be a favorable target of the seismological studies especially on low-mass stars. Then, the seismic modes of the acoustic eigen-frequencies will provide more stringent constraints that define the internal structure of the star.
Preferentially, the $p$-mode spectrum can be characterized by the large frequency separation which is the inverse of the sound travel time (going and returning) from the surface to the core, that is,

$$
\Delta \nu \equiv\left(2 \int \frac{d r}{c(r)}\right)^{-1}
$$

where $c(r)$ is the sound speed at the radius $r$ inside stars. Therefore, the large separation is very sensitive to the radius (as a boundary condition) and the density profile (as a function of the depth) inside the star (Aerts et al. 2010). If the mode frequencies are detected, then it can be inversely translated to the density profile. For the low degree $p$-modes, the large separation can be written as

$$
\Delta \nu=\nu_{n+1, l}-\nu_{n, l},
$$

and reduced frequency modulo is

$$
\nu_{n, l}=\nu_{0}+k \Delta \nu+\tilde{\nu}_{n, l},
$$

where $\nu_{0}$ is a suitably chosen reference frequency, and $k$ is an integer such that $\tilde{\nu}_{n l}$ lies between 0 and $\Delta \nu$. According to the asymptotic expansion (Gough 1986), the small frequency separation can be defined as

$$
\begin{aligned}
\delta \nu_{n, l} & \equiv \nu_{n, l}-\nu_{n-1, l+2} \\
& \simeq-(4 l+6) \frac{\Delta \nu}{4 \pi^{2} \nu_{n, l}} \int_{0}^{R} \frac{d c(r)}{d r} \frac{d r}{r} .
\end{aligned}
$$

This implies that the small frequency separation is seriously affected by the sound-speed gradient in the core. In other words, it is closely related to the chemical composition of the core, that is, the evolutionary status. On the basis of the non-adiabatic analysis (Guenther 1994), the theoretical frequency spectrum is computed using the best fit model of $\mu$ Cas A. In the left panel of Figure 4 , the large frequency separations $\Delta \nu$ of two modes $(l=0,2)$ are presented. Two kinds of the small frequency separations $\left(\delta \nu_{n, 0}, \delta \nu_{n, 1}\right)$ are calculated in the right panel. In addition, the Echelle diagram of the frequency spectrum is illustrated in Figure 5. Circles, triangles, squares, and diamonds correspond to modes $l=0,1,2$, and 3 , respectively. From our seismic computations, we find that $\mu$ Cas $\mathrm{A}$ has the mean first order spacing $\langle\Delta \nu\rangle=169 \mu \mathrm{Hz}$ with the reference frequency $\nu_{0}=120 \mu \mathrm{Hz}$. If the mode frequencies can be detected, these models will provide a more stringent constraint of the evolutionary status of $\mu$ Cas.

\section{Discussion AND SUmmary}

An elemental enhancement in abundances of metalpoor stars plays a key role in probes of the formation history of the Galaxy. In particular, the mutual connection between $[\alpha / \mathrm{Fe}]$ and $[\mathrm{Fe} / \mathrm{H}]$ can be understood from a point of the stellar nucleosynthesis feed-back to the interstellar medium. Therefore, it is worthwhile to discuss the correlations among the stellar parameters such as metallicity, $\alpha$-enhancement, and space velocity. From its chemical characteristics $([\alpha / \mathrm{Fe}] \sim+0.4$ 

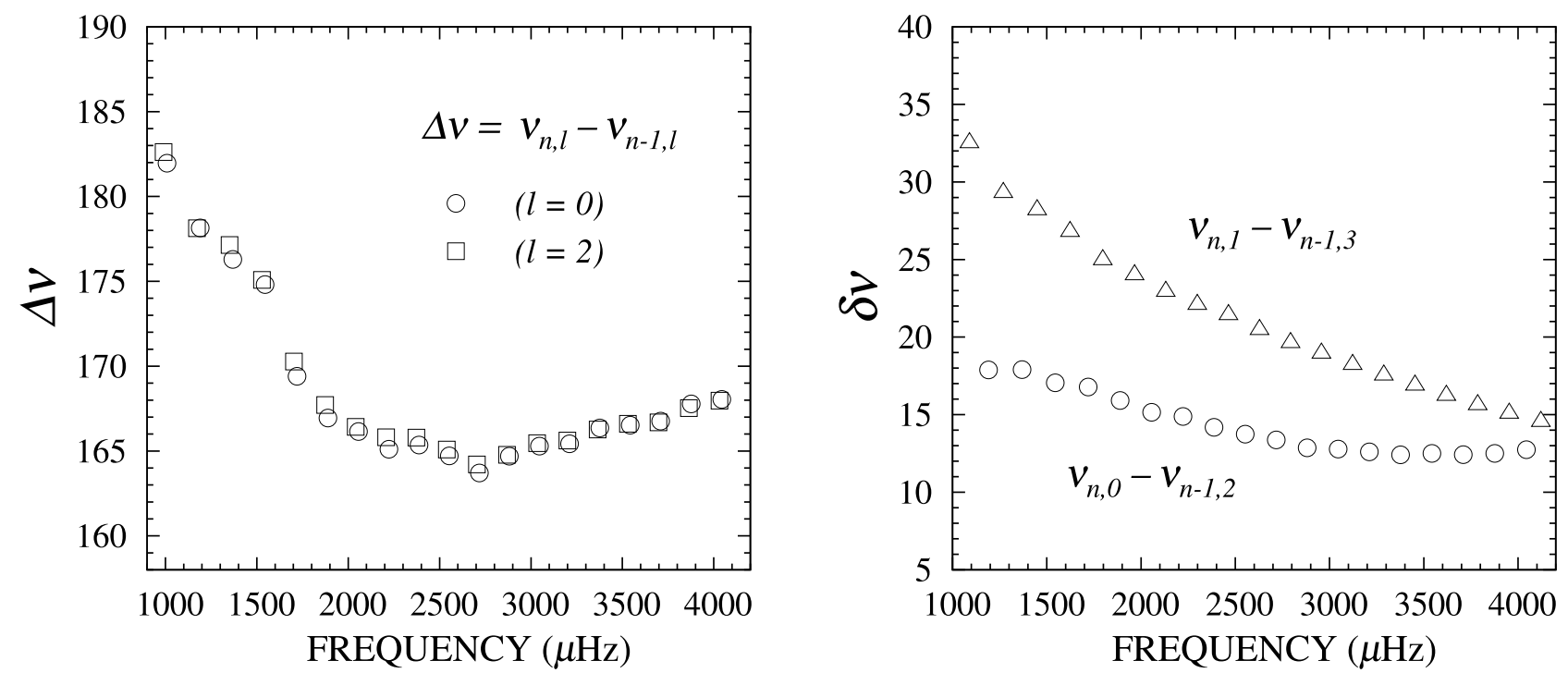

Figure 4. Theoretical estimations of the $p$-mode frequencies of the primary component of $\mu$ Cas. In the context of the asteroseismology, the large frequency separations $\Delta \nu$ (left) and the small frequency separations $\delta \nu($ right) are calculated. Circles donote $l=0$ modes, triangles $l=1$ modes, and squares $l=2$ modes.

$\operatorname{dex},[\mathrm{Fe} / \mathrm{H}] \sim-0.8 \mathrm{dex}), \mu$ Cas seems to be categorized not into a typical halo star $([\mathrm{Fe} / \mathrm{H}]<-1)$ but into a thick disk star (Bensby \& Feltzing 2006; Reddy et al. 2006; Mishenina et al. 2013). Moreover, the space velocity with respect to the local standard of rest $\left(V_{L S R} \sim 160 \mathrm{~km} \mathrm{~s}^{-1}\right)$ belongs to the thick disk domain $\left(V_{L S R} \gtrsim 182 \mathrm{~km} \mathrm{~s}^{-1}\right)$ (Takeda 2007). In addition, the maximum separation from the Galactic plane $\left(z_{\max } \sim 0.35 \mathrm{kpc}\right)$ also corresponds to an intermediate value of disk stars $\left(z_{\max }<0.5\right)$ (Ibukiyama \& Arimoto 2002; Takeda 2007). For these reasons, $\mu$ Cas seems to be marginally close to the thick disk stars.

In stellar astrophysics, the surface convection is one of the most uncertain physical process. Inevitably, in the 1D stellar models, convection phenomenon has been treated by the classical mixing length parameter (Böhm-Vitense 1958). Within a range of mild variations from the solar value, our model computation of $\mu$ Cas includes calibrations of the mixing length parameter. Conventionally, the well-defined binary stars provide a good estimation of the mixing length parameters in a semi-empirical way. For instance, the $\alpha$ Cen binary system is one of the closest multiple systems whose physical dimensions are well-defined (Demarque et al. 1986; Kim 1999). In the recent studies, it was calibrated to be $\alpha_{A} \sim 1.64-1.86$ and $\alpha_{B} \sim 1.58-1.97$ (Morel et al. 2000; Yildiz 2007). In the case of $85 \mathrm{Peg}$, the mixing length parameters are known to have $\left(\alpha_{A}, \alpha_{B}\right)=$ $(1.85,2.14)$ by Fernandes et al. $(2002)$, and $\left(\alpha_{A}, \alpha_{B}\right)=$ $(1.6,1.4)$ by Bach et al. (2009). Generally, many stellar evolution codes employ a different model atmospheres and physical inputs, which leads to a slight change in optical and thermal properties near the surface, and therefore the mixing length parameters are calibrated into different values. However, the calibrated mixing length parameters are still closed to their solar value $\alpha_{\odot}$ generated from the standard solar model (Trampedach et al. 2014b).

Indeed, to improve estimations of the mixing length parameters, stellar model atmosphere as an outer boundary condition should be defined priorly. The majority of stellar models adopt the grey atmosphere approximation (Mihalas 1978) which relates the temperature distribution to the mean optical depth $\tau$ in the outer layers. This is the well-known the Eddington $T-\tau$ relation, and it can be generalized by

$$
T(\tau)^{4}=\frac{3}{4} T_{\mathrm{eff}}^{4}[\tau+q(\tau)]
$$

with the Hopf function $(q(\tau) \simeq 2 / 3)$ that is originally introduced to describe radiative transfer in a semiinfinite atmosphere (Hopf 1930).

For the solar-like star whose outer region is covered with the envelope, the optical transmittance is seriously affected by the large temperature fluctuation driven by convection. The surface convection zone is an extremely turbulent region where the partly ionized particles move fast at high temperature. In particular, the superadiabatic layers (SAL) are a transition region where the transport of energy immediately changes from convection to radiation. In order to describe radiant flows, a detailed treatment of radiative transfer (RT) should be included in the hydrodynamic (HD) simulations.

Recent advances in numerical simulations have enabled to directly compute the surface convection in 3D radiation-hydrodynamics (RHD) (Chan \& Sofia 1987; Stein \& Nordlund 1989; Freytag et al. 1996). From their comparison test of the solar model, Bach \& Kim (2012) found that the location of the SAL in the 3D RHD model considerably differs from those of the 1D model due to turbulent motions of the fully compressible flows. Furthermore, recent measurements of the solar abundances based on the 3D RHD simulations (Asplund et al. 2005, 2009) seriously differs from the 


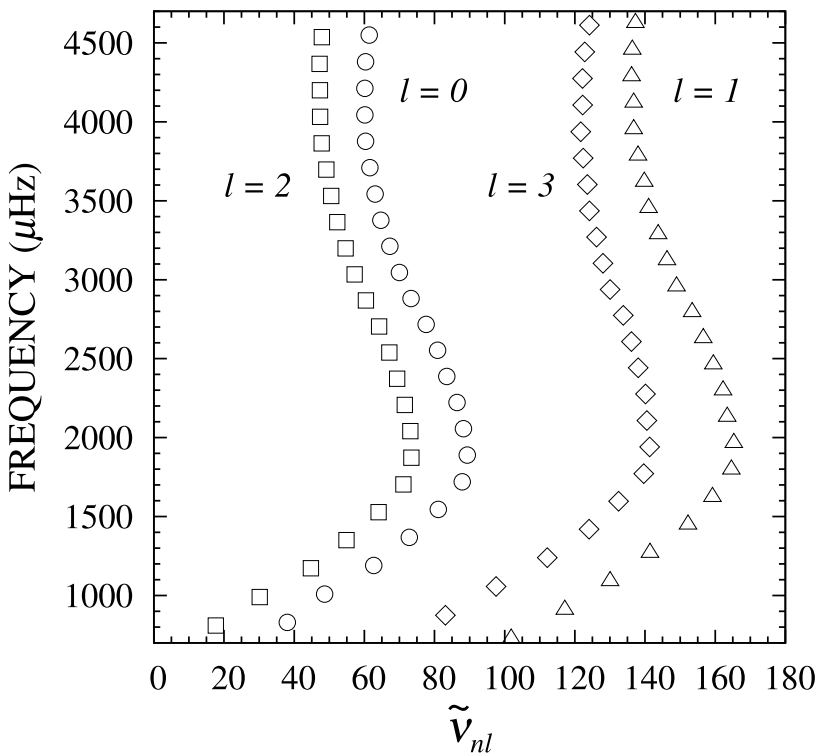

Figure 5. The Echelle diagram of the frequency spectrum for our model of $\mu$ Cas is presented. With our calibrated parameters, the theoretical frequency spectrum was calculated in the context of asteroseismology. Circles, triangles, squares, and diamonds correspond to modes $l=0,1,2$, and 3 , respectively. The approximated large separation is $\Delta \nu \sim 169 \mu \mathrm{Hz}$ at the reference frequency $\nu_{0}=120 \mu \mathrm{Hz}$.

previous one (Grevesse \& Sauval 1998). More recently, through the intensive RHD simulations (Trampedach et al. 2014a,b; Tanner et al. 2014), they attempted to evaluate the validity of the classical approximations. Especially, Trampedach et al. (2014b) provided an absolute calibration of the mixing length parameter combined with a realistic $T-\tau$ relation considering a wide range of the evolutionary stage. In addition, Tanner et al. (2014) examined the effect of metallicity on convection and overshoot in the SAL of main-sequence stars. However, such an inclusion of the detailed radiative transfer in hydrodynamic simulations is still computationally expensive. In order to quantify the classical approximations, a larger variety of model grids (metalpoor, low-mass) should be counted. In this respect, based on a well-defined internal structure of $\mu$ Cas, we are computing the 3D RHD model, which will provide more detailed information about the surface convection phenomena of the metal-poor low-mass stars. On the other hand, using the well-defined stellar parameters of nearby stars, (Chen et al. 2014) provided an empirically calibrated $T-\tau$ relation for the very low-mass stars (VLMS). They found that the newly determined $T-\tau$ relation has a significant effect on the stellar radii (larger) and temperature (cooler) for the lower MS star. The secondary star $\mu$ Cas B is regarded as a very lowmass star $\left(M_{B} \sim 0.2 \mathrm{M}_{\odot}\right)$, and our main-sequence solution is calibrated into a slightly higher temperature than the center value of observational error. If a newly calibrated $T-\tau$ relation is applied to our computation, a more accurate modelling will be possible.

Based on the high-resolution spectroscopy of the
BOES observation (Bach \& Kang 2015), we investigate physical properties of the $\mu$ Cas binary system. By combining recent observations, stellar parameters of this binary system have been calibrated in the context of standard stellar theory. In our evolutionary computation, the whole physical inputs such as opacities and the equation of state are consistently generated based on the chemical mixture $([\alpha / \mathrm{Fe}] \gtrsim+0.4 \mathrm{dex}$, $[\mathrm{Fe} / \mathrm{H}] \sim-0.7 \mathrm{dex})$. Through the statistical evaluation of the $\chi^{2}$-minimization among alternative models, we find a reliable evolutionary solution $\left(M_{A}, M_{B}, t_{\text {age }}\right)=$ $\left(0.74 \mathrm{M}_{\odot}, 0.19 \mathrm{M}_{\odot}, 11 \mathrm{Gyr}\right)$ which excellently satisfies observational constraints. In particular, we confirm that the initial helium abundance of $\mu$ Cas is comparable to the primordial helium contents $\left(Y_{p} \sim 0.245\right)$. From the best fit model of the primary star, the $p$ mode frequencies are estimated within the framework of asteroseismology. With the well-defined stellar parameters, $\mu$ Cas will provide deeper insight of various physical processes inside the metal-poor low-mass stars.

\section{ACKNOWLEDGMENTS}

We are grateful to Yong-Cheol Kim, Pierre Demarque, and Marc Pinsonneault for their insightful discussions on physical processes of stellar astrophysics. The author sincerely appreciates Young-Woon Kang and JaeWoo Lee for their invaluable discussions on the binary system and spectroscopic analysis. The author thanks Jason W. Ferguson for his opacity computations at low temperature. We also thank Evangelia Tremou and Camilla Pacifici for their helpful comments on a draft version of this paper. This research was supported by the BK21 Plus Research Programme (21A-201315-00002) and in part by the Basic Science Research Programme (2014-11-1019) through the National Research Foundation (NRF) funded by the Ministry of Education. The author gratefully acknowledges support of computing facilities of Yonsei Observatory and the KASI-Yonsei DRC programme of Korea Research Council of Fundamental Science and Technology (DRC12-2-KASI).

\section{REFERENCES}

Aerts, C., Christensen-Dalsgaard, J., \& Kurtz, D. W. 2010, Asteroseismology, Astronomy and Astrophysics Library (Berlin: Springer)

Alonso, A., Arribas, S., \& Martínez-Roger, C. 1999, The Effective Temperature Scale of Giant Stars (F0-K5). II. Empirical Calibration of $T_{\text {eff }}$ versus Colours and $[\mathrm{Fe} / \mathrm{H}]$, A\&AS, 140, 261

Asplund, M., Grevesse, N., \& Sauval, A. J. 2005, The Solar Chemical Composition, ASPC, 336, 25

Asplund, M., Grevesse, N., Sauval, A. J., \& Scott, P. 2009, The Chemical Composition of the Sun, ARA\&A, 47, 481

Bach, K., Lee, J., Demarque, P., \& Kim, Y.-C. 2009, Evolutionary Status of 85 Pegasi, ApJ, 703, 362

Bach, K., \& Kim, Y.-C. 2012, Hydrodynamical Comparison Test of Solar Models, Astron. Nachr., 333, 934

Bach, K., \& Kang, W. 2015, Determination of Physical Dimensions of $\mu$ Cas, ASPC, in press 
Bahcall, J. N., \& Loeb, A. 1990, Element Diffusion in Stellar Interiors, ApJ, 360, 267

Basu, S., \& Antia, H. M. 2008, Helioseismology and Solar Abundances, PhR, 457, 217

Bensby, T., \& Feltzing, S. 2006, The Origin and Chemical Evolution of Carbon in the Galactic Thin and Thick Discs, MNRAS, 367, 1181

Berger, D. H., Gies, D. R., McAlister, H. A., et al. 2006, First Results from the CHARA Array. IV. The Interferometric Radii of Low-Mass Stars, ApJ, 644, 475

Böhm-Vitense, E. 1958, Über die Wasserstoffkonvektionszone in Sternen Verschiedener Effektivtemperaturen und Leuchtkräfte. Mit 5 Textabbildungen, Z. Astrophys., 46, 108

Boyajian, T. S., McAlister, H. A., Baines, E. K., et al. 2008, Angular Diameters of the G Subdwarf $\mu$ Cassiopeiae A and the K Dwarfs $\sigma$ Draconis and HR 511 from Interferometric Measurements with the CHARA Array, ApJ, 683, 424

Caloi, V., Cardini, D., D'Antona, F., Badiali, M., Emanuele, A., \& Mazzitelli, I. 1999, Kinematics and Age of Stellar Populations in the Solar Neighbourhood from Hipparcos Data, A\&A, 351, 925

Cassisi, S., Salaris, M., \& Irwin, A. W. 2003, The Initial Helium Content of Galactic Globular Cluster Stars from the R-Parameter: Comparison with the Cosmic Microwave Background Constraint, ApJ, 588, 862

Catchpole, R. M., Pagel, B. E. J., \& Powell, A. L. T. 1967, Chemical Composition of the Mild Subdwarf $\mu$ Cassiopeiae, MNRAS, 136, 403

Chan, K. L., \& Sofia, S. 1987, Validity Tests of the MixingLength Theory of Deep Convection, Sci., 235, 465

Chen, Y., Girardi, L., Bressan, A., Marigo, P., Barbieri, M., \& Kong, X./ 2014, Improving PARSEC Models for Very Low Mass Stars, MNRAS, 444, 2525

Chieffi, A., Straniero, O., \& Salaris, M. 1995, Calibration of Stellar Models, ApJ, 445, 39

Cohen, J. G. 1968, Analysis of F and G Subdwarfs. III. an Abundance Analysis of the Subdwarf $\mu$ Cassiopeia, ApJ, 154,179

Cox, J. P., \& Giuli, R. T. 1968, Principles of Stellar Structure (NewYork: Gordon \& Breach)

Demarque, P., Guenther, D. B., \& van Altena, W. F. 1986, The Case of Alpha Centauri - Mass, Age and P-Mode Oscillation Spectrum, ApJ, 300, 773

Dennis, T. R. 1965, On the Possibility of Determining the Helium Content of the Subdwarf $\mu$ Cassiopeiae, PASP, 77,283

Drummond, J. D., Christou, J. C., \& Fugate, R. Q. 1995, Full Adaptive Optics Images of ADS 9731 and $\mu$ Cassiopeiae: Orbits and Masses, ApJ, 450, 380

Eggen, O. J., Lynden-Bell, D., \& Sandage, A. R. 1962, Evidence from the motions of old stars that the Galaxy collapsed, ApJ, 136, 748

Feibelman, W. A. 1976, The Astrometric Binary MU Cassiopeiae - Photographically Almost Resolved, ApJ, 209, 497

Feltzing, S., \& Gustafsson, B. 1998, Abundances in MetalRich Stars. Detailed Abundance Analysis of $47 \mathrm{G}$ and $\mathrm{K}$ Dwarf Stars with $[\mathrm{Me} / \mathrm{H}]>0.10$ dex, A\&AS, 129, 237

Ferguson, J. W., Alexander, D. R., Allard, F., et al. 2005, Low-Temperature Opacities, ApJ, 623, 585

Fernandes, J., Lebreton, Y., Baglin, A., \& Morel, P. 1998, Fundamental Stellar Parameters for Nearby Visual Binary Stars : $\eta$ Cas, XI Boo, $70 \mathrm{OPH}$ and 85 Peg. Helium
Abundance, Age and Mixing Length Parameter for Low Mass Stars, A\&A, 338, 455

Fernandes, J., Morel, P., \& Lebreton, Y. 2002, A Calibration of the 85 Peg Binary System, A\&A, 392, 529

Fuhrmann, K. 1998, Surface Gravities of Very Metal-Poor Stars from HIPPARCOS Parallaxes, A\&A, 330, 626

Fuhrmann, K. 1998, Nearby Stars of the Galactic Disk and Halo, A\&A, 338, 161

Fulbright, J. P. 2000, Abundances and Kinematics of Field Halo and Disk Stars. I. Observational Data and Abundance Analysis, AJ, 120, 1841

Freytag, B., Ludwig, H.-G., \& Steffen, M. 1996 Hydrodynamical Models of Stellar Convection. The Role of Overshoot in DA White Dwarfs, A-Type Stars, and the Sun, A\&A, 313, 497

Gamow, G. 1948, The Origin of Elements and the Separation of Galaxies, Phys. Rev., 74, 505

Gough, D. O., 1986, Asymptotic Sound-Speed Inversions, ASIC, 169, 125

Grevesse, N., \& Sauval, A. J. 1998, Standard Solar Composition, SSRv, 85, 161

Guenther, D. B., Demarque, P., Kim, Y.-C., \& Pinsonneault, M. H. 1992, Standard Solar Model, ApJ, 387, 372

Guenther, D. B. 1994, Nonadiabatic Nonradial p-mode Frequencies of the Standard Solar Model, with and without Helium Diffusion, ApJ, 422, 400

Haywood, J. W., Hegyi, D. J., \& Gudehus, D. H., 1992, A Measurement of the Primordial Helium Abundance Using $\mu$ Cassiopeiae, ApJ, 392, 172

Hearnshaw, J. B. 1974, Carbon and Iron Abundances for Thirty F and G Type Stars, A\&A, 34, 263

Hopf, E. 1930, Remarks on the Schwarzschild-Milne Model of the Outer Layers of a Star, MNRAS, 90, 287

Ibukiyama, A., \& Arimoto, N. 2002, HIPPARCOS AgeMetallicity Relation of the Solar Neighbourhood Disc Stars, A\&A, 394, 927

Iglesias, C. A., \& Rogers, F. J. 1996, Updated Opal Opacities, ApJ, 464, 943

Israelian, G., García López, R. J., \& Rebolo, R. 1998, Oxygen Abundances in Unevolved Metal-poor Stars from Near-Ultraviolet OH Lines, ApJ, 507, 805

Izotov, Y. I., \& Thuan, T. X. 2010 The Primordial Abundance of ${ }^{4} \mathrm{He}$ : Evidence for Non-Standard Big Bang Nucleosynthesis, ApJ, 710, 67

Kim, K.-M., Jang, B.-H., Han, I., Jang, J. G., et al. 2002, Design and Manufacturing of the Cassegrain Interface Module of the BOAO Echelle Spectrograph, JKAS, 35, 221

Kim, Y.-C. 1999, Standard Stellar Models; $\alpha$ Cen A and B, JKAS, 32, 119

Lane, B. F., Boden, A. F., \& Kulkarni, S. R. 2001, Interferometric Measurement of the Angular Sizes of Dwarf Stars in the Spectral Range K3-M4, ApJ, 551, 81

Lastennet, E., Valls-Gabaud, D., Lejeune, Th., \& Oblak, E. 1999, Consequences of HIPPARCOS Parallaxes for Stellar Evolutionary Models. Three Hyades binaries: V 818 Tauri, 51 Tauri, and $\theta(2)$ Tauri, A\&A, 349, 485

Lebreton, Y., Perrin, M.-N., Cayrel, R., Baglin, A., \& Fernandes, J. 1999, The HIPPARCOS HR Diagram of Nearby Stars in the Metallicity Range: $-1.0<[\mathrm{Fe} / \mathrm{H}]<$ 0.3. A New Constraint on the Theory of Stellar Interiors and Model Atmospheres, A\&A, 350, 587

Lippincott, S. L., \& Wyckoff, S. 1964, Parallax and Orbital Motion of the Astrometric Binary $\mu$ Cassiopeiae from Photographs Taken with the 24-inch Sproul Refractor, 
AJ, 69, 471

Lippincott, S. L. 1981, Astrometric Analysis of the Unresolved Binary $\mu$ Cassiopeiae from Photographs Taken with the Sproul 61 Centimeter Refractor, ApJ, 248, 1053

McCarthy, D. W. Jr., Hancock, T., Freeman, J., et al. 1993, Infrared imaging of $\mu$ CAS B using rapid image motion compensation, AJ, 105, 652

Meléndez, J. and Barbuy, B., \& Spite, F. 2001, Oxygen Abundances in Metal-poor Stars $(-2.2<[\mathrm{Fe} / \mathrm{H}]<-1.2)$ from Infrared OH Lines, ApJ, 556, 858

Mihalas, D. 1978, Stellar Atmospheres 2nd edn. (San Francisco: Freeman and Co.)

Mishenina, T. V., Kovtyukh, V. V., Soubiran, C., Travaglio, C., \& Busso, M. 2002, Abundances of $\mathrm{Cu}$ and $\mathrm{Zn}$ in MetalPoor Stars: Clues for Galaxy Evolution, A\&A, 396, 189

Mishenina, T. V., Soubiran, C., Kovtyukh, V. V., \& Korotin, S. A. 2004, On the Correlation of Elemental Abundances with Kinematics among Galactic Disk Stars, A\&A, 418, 551

Mishenina, T. V., Pignatari, M., Korotin, S. A., et al. 2013, Abundances of Neutron-Capture Elements in Stars of the Galactic Disk Substructures, A\&A, 552A, 128

Morel, P., Provost, J., Lebreton, Y., Thévenin, F., \& Berthomieu, G. 2000, Calibrations of $\alpha$ Centauri A \& B, A\&A, 363, 675

Nissen, P. E., Hoeg, E., \& Schuster, W. J. 1997, Surface Gravities of Metal-Poor Stars Derived from HIPPARCOS Parallaxes, in Proc. of the ESA Symp., HIPPARCOS Venice 97, ed. B. Battrick (ESA SP-402; Noordwijk: ESA), 225

Pagel, B. E. J. 2009, Nucleosynthesis and Chemical Evolution of Galaxies (Cambridge: Cambridge University Press)

Peebles, P. J. E. 1966 Primordial Helium Abundance and the Primordial Fireball. II, ApJ, 146, 542

Peimbert, M., \& Torres-Peimbert, S. 1999, Peebles's Analysis of the Primordial Fireball, ApJ Centennial Issue, 525, 1143

Peimbert, M., Luridiana, V., \& Peimbert, A. 2007, Revised Primordial Helium Abundance Based on New Atomic Data, ApJ, 666, 636

Reddy, B. E., Lambert, D. L., \& Allende Prieto, C. 2006, Elemental Abundance Survey of the Galactic Thick Disc, MNRAS, 367, 1329

Ribas, I., Jordi, C., Torra, J., \& Giménez, Á. 2000, Chemical Composition of Eclipsing Binaries: A New Approach to the Helium-to-Metal Enrichment Ratio, MNRAS, 313, 99

Russell, J. L., \& Gatewood, G. D. 1984, The Parallax and Astrometric Orbit of $\mu$ Cassiopeiae, PASP, 96, 429

Ségransan, D., Kervella, P., Forveille, T., \& Queloz, D. 2003, First Radius Measurements of Very Low Mass Stars with the VLTI, A\&A, 397L, 5S

Steigman, G. 2007, Primordial Nucleosynthesis in the Precision Cosmology Era, ARNPS, 57, 463
Stein, R. F., \& Nordlund, Å. 1989, Topology of Convection Beneath the Solar Surface, ApJ, 342, 95

Takeda, Y., \& Honda, S. 2005, Photospheric CNO Abundances of Solar-Type Stars, PASJ, 57, 65

Takeda, Y. 2007, Fundamental Parameters and Elemental Abundances of 160 F-G-K Stars Based on OAO Spectrum Database, PASJ, 59, 335

Takeda, Y., \& Takada-Hidai, M. 2011, Exploring the [S/Fe] Behavior of Metal-Poor Stars with the Si $1.046 \mu \mathrm{m}$ Lines, PASJ, 63S, 537

Tanner, J. D., Basu, S., \& Demarque, P. 2014, The Effect of Metallicity-dependent T-tau Relations on Calibrated Stellar Models, ApJ, 785, 13

Thévenin, F., \& Idiart, T. P. 1999, Stellar Iron Abundances: Non-LTE Effects, ApJ, 521, 753

Thoul, A. A., Bahcall, J. N., \& Loeb, A,. 1994 Element Diffusion in the Solar Interior, ApJ, 421, 828

Trampedach, R., Stein, R. F., Christensen-Dalsgaard, J., Nordlund, Å, \& Asplund, M. 2014, Improvements to stellar structure models, based on a grid of $3 \mathrm{D}$ convection simulations - I. T(tau) relations, MNRAS, 442, 805

Trampedach, R., Stein, R. F., Christensen-Dalsgaard, J., Nordlund, A, \& Asplund, M. 2014, Improvements to stellar structure models, based on a grid of $3 \mathrm{D}$ convection simulations - II. Calibrating the mixing-length formulation, MNRAS, 445, 4366

Vandenberg, D. A., \& Bridges, T. J. 1984, Theoretical Zeroage Main Sequences Applied to the Pleiades, Praesepe, and Hyades Star Clusters, ApJ, 278, 679

Vandenberg, D. A., \& Hrivnak, B. J. 1985, The Age and Helium Content of the Eclipsing Binary AI Phoenicis, ApJ, 291, 270

Villanova, S., Piotto, G., \& Gratton, R. G. 2009, The Helium Content of Globular Clusters: Light Element Abundance Correlations and HB Morphology. I. NGC 6752, A\&A, 499, 755

Wagman, N. E. 1961, Reports of Observatories, AJ, 66, 433

Wagman, N. E., Daniel, Z., \& Crissman, B. G. 1963, Photographic Determinations of the Parallaxes of 60 stars with the Thaw Refractor, AJ, 68, 352

Weinberg, S. 2008, Cosmology (Oxford: Oxford University Press)

Wickes, W. C., \& Dicke, R. H. 1974, Achromatic Doublestar interferometry, AJ, 79, 1433

Wickes, W. C. 1975, Interferometric Measurements of Binary Stars, AJ, 80, 655

Worek, T. F., \& Beardsley, W. R. 1977, A Spectroscopic Orbit for the Subdwarf Binary $\mu$ Cassiopeiae, ApJ, 217, 134

Yildiz, M. 2007, Models of $\alpha$ Centauri A and B With and Without Seismic Constraints: Time Dependence of the Mixing-Length Parameter, MNRAS, 374, 1264

Zhao, G., \& Gehren, T. 2000, Non-LTE Analysis of Neutral Magnesium in Cool Stars, A\&A, 362, 1077 